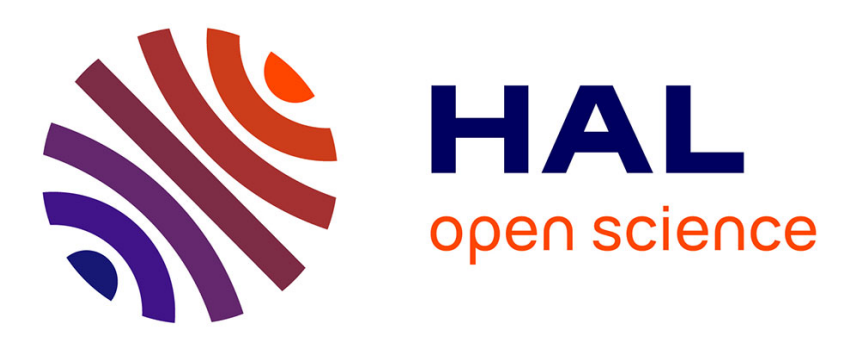

\title{
Networked Conflicting Timed Event Graphs Representation in (Max, +) Algebra
}

Boussad Addad, Saïd Amari, Jean-Jacques Lesage

\section{To cite this version:}

Boussad Addad, Saïd Amari, Jean-Jacques Lesage. Networked Conflicting Timed Event Graphs Representation in $(\mathrm{Max},+)$ Algebra. Discrete Event Dynamic Systems, 2012, 22 (4), pp.429-449. hal00782849

\section{HAL Id: hal-00782849 \\ https://hal.science/hal-00782849}

Submitted on 31 Jan 2013

HAL is a multi-disciplinary open access archive for the deposit and dissemination of scientific research documents, whether they are published or not. The documents may come from teaching and research institutions in France or abroad, or from public or private research centers.
L'archive ouverte pluridisciplinaire HAL, est destinée au dépôt et à la diffusion de documents scientifiques de niveau recherche, publiés ou non, émanant des établissements d'enseignement et de recherche français ou étrangers, des laboratoires publics ou privés. 


\title{
Networked Conflicting Timed Event Graphs Representation in (Max,+) Algebra
}

\author{
ADDAD, B.*, AMARI, S.*, LESAGE, J-J.* \\ *Automated Production Research Laboratory (LURPA), 61 av du President \\ Wilson, Cachan 94235 France, \\ (Tel: (+33) 01474027 62; e-mail: Boussad.addad@lurpa.ens-cachan.fr, \\ said.amari@lurpa.ens-cachan.fr,Jean-jacques.lesage@lurpa.ens-cachan.fr).
}

\begin{abstract}
Timed Event Graphs (TEGs) are a specific class of Petri nets that have been thoroughly studied given their useful linear state representation in $(\mathrm{Max},+)$ algebra. Unfortunately, TEGs are generally not suitable for modeling systems displaying resources sharing (or conflicts). In this paper, we show that if a system with conflicts is modeled using a NCTEG (Networked Conflicting Timed Event Graphs), it is quite possible to obtain an equivalent $(\mathrm{Max},+)$ representation. More precisely, we prove that the evolution of a NCTEG satisfies linear time-varying $(\mathrm{Max},+)$ equations. In case of cyclic NCTEGs, which are a natural model of many repetitive systems, we provide a standard time-invariant $(\mathrm{Max},+)$ representation. As an application of the proposed approach to exhibit its interest, we consider the case of Jobshops. We first propose a generic NCTEG-based model of these systems and subsequently apply the corresponding (Max, + ) representation to evaluate some of their performances.
\end{abstract}

Keywords: Timed Event Graph, (Max,+) algebra, Conflict, Linear-Time Varying

Systems, Repetitive Systems.

\section{INTRODUCTION}

Petri nets are a powerful tool for discrete events systems modeling and analysis. They are often used to represent phenomena like synchronization, parallelism and concurrency (Murata, 1989). Their domain of application is very large, including manufacturing systems, communication systems, transportation, etc. Many classes of Petri nets with more or less elaborated semantics are used according to the context of the study. Generally speaking, the more their structure and semantics are elaborated the more complex is their analysis. The relatively simple class of Petri nets called Timed Event Graphs (TEGs) is likely to be the most investigated one. Indeed, TEGs are easily represented in the form of linear equations in $(\mathrm{Max},+)$ algebra, provided that the places and the transitions be overtaking free (FIFO places and FIFO transitions as defined in Baccelli et al. (1992)). This linear $(\mathrm{Max},+)$ form being very similar to the state representation of the classical discrete linear systems, the main related results are mostly straightforwardly applied (Cohen et al. 1999). Unfortunately, TEGs are Petri nets with places displaying at most one upstream transition and one downstream transition. Thus, 
they are not suitable for modeling systems with resources sharing or conflicts. Note nonetheless that some particular systems involving shared resources (e.g. flowshops), can be modelled using TEGs with time-varying parameters (Lahaye et al., 2004). The authors had however to change the usual FIFO rule to prevent tokens from overtaking and finally to get to linear $(\mathrm{Max},+)$ equations. Such a consideration is possible in some cases but it is unfortunately not always the case. In literature, a number of other efforts have been undertaken to tackle differently the problem of conflicts. The authors in Hillion et al. (1989) investigated the problem of repetitive systems i.e. with a cyclic allocation of the shared resources. They proposed to transform the original Petri net with conflicts into a TEG, the $(\mathrm{Max},+)$ representation of this latter being easily obtained. In Gaubert et al. (1999), an algebraic modeling, based on the heaps-of-pieces theory and (Max,+) automata, is provided for safe Petri nets. For the case of Free Choice Petri nets, a very complete analytic study is proposed in Baccelli et al. (1996). The case of processes that switch between different functioning modes is investigated in Van Den Boom et al. (2006) using switching (Max,+) linear systems. We can also quote Correïa et al. (2009) where local $(\mathrm{Max},+)$ equations are written without taking into account the conflicts. A constraint (inequality) is then added to represent only the admissible evolutions of the global system. In Nait et al. (2006), a method introducing the concept of virtual firing of transitions is proposed for a transportation system. More recently (Boutin et al., 2009), an approach based on the dioid of intervals is used to represent extreme behaviors of a manufacturing system (with shared resources i.e. conflicts context) and provide the bounds of its production rate.

In the current investigation, we propose a novel approach to model a large category of systems involving shared resources. This paper intends to extend the study proposed in Addad et al. (2010) concerning Networked Conflicting Timed Event Graphs (NCTEGs) and their $(\mathrm{Max},+)$ representation. Moreover, it provides a generic NCTEG-based model of the well-known systems called jobshops. In a more general context, the purpose of our investigation was originally to extend the use of $(\mathrm{Max},+)$ algebra to the class of NCTEGs which are much larger than TEGs. On top of that, some of the previously exposed hypotheses in the existing works about conflicts are relaxed:

i) the TEGs that constitute the NCTEG are not necessarily safe (they, by the way, display input transitions).

ii) the conflicting transitions can have more than one upstream place (unlike Free Choice Petri nets).

iii) resources allocation policy is not necessarily cyclic.

The remainder of this paper is organized as follows: Section 2 recalls some basic notions about TEGs and their linear $(\mathrm{Max},+)$ representation. Section 3 is dedicated to the study of NCTEGs in $(\mathrm{Max},+)$ algebra: Section 3.1 introduces some definitions and notations with regard to NCTEGs and Section 3.2 presents their modeling using $(\mathrm{Max},+)$ equations. Thereafter, a linear time-varying reformulation is provided in Section 4. Hence, a standard time-invariant linear state form is provided for cyclic NCTEGs in Section 5. Then, Section 6 exposes jobshops as an application of NCTEGs and their $(\mathrm{Max},+)$ representation. Finally, Section 7 concludes this paper with some outlooks for future work. 


\section{Linear $($ Max,+) equations of TEGs}

In this section, we recall the linear $(\mathrm{Max},+)$ representation of TEGs that will be useful in the sequel of this paper. Note that a mix of t-timed and p-timed TEGs are considered in our study. If their delays are not depicted, they are supposed to be null. Let us consider the simple example below (a t-timed one).

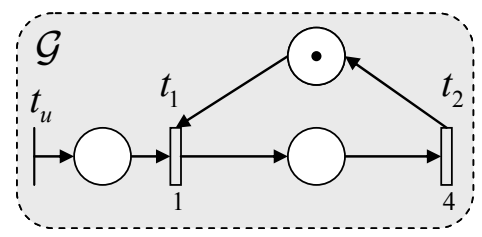

Fig. 1. Example of TEG.

Let $x_{i}(k), i=1,2$ (resp. $u(k)$ ) be the date of firing transition $t_{i}$ (resp. $t_{u}$ ) for the $k^{\text {th }}$ time. If firing is performed at maximal speed (as soon as possible), we can write the equations:

$$
\left\{\begin{array}{l}
x_{1}(k)=1+\max \left(u(k), x_{2}(k-1)\right) \\
x_{2}(k)=4+x_{1}(k)
\end{array}\right.
$$

The equations above can be rewritten using $(\mathrm{Max},+)$ algebra operators: the classical maximum noted $\oplus$ and the usual addition noted $\otimes$. These operators are defined on the set $\mathbb{R}_{\max }=\mathbb{R} \cup\{-\infty\}$ and have respectively $\varepsilon=-\infty$ and $e=0$ as null and identity elements. Equations (1) become:

$$
\left\{\begin{array}{l}
x_{1}(k)=1 \otimes u(k) \oplus 1 \otimes x_{2}(k-1) \\
x_{2}(k)=4 \otimes x_{1}(k)
\end{array}\right.
$$

Using a matrix notation, we finally get to:

$$
X(k)=A_{0} \otimes X(k) \oplus A_{1} \otimes X(k-1) \oplus B_{0} \otimes U(k)
$$

with: $X=\left(\begin{array}{ll}x_{1} & x_{2}\end{array}\right)^{t}, U=u, A_{0}=\left(\begin{array}{ll}\varepsilon & \varepsilon \\ 4 & \varepsilon\end{array}\right), A_{1}=\left(\begin{array}{ll}\varepsilon & 1 \\ \varepsilon & \varepsilon\end{array}\right)$, and $B_{0}=\left(\begin{array}{l}1 \\ \varepsilon\end{array}\right)$.

Equation (3) can also be brought to an explicit form thanks to the following theorem:

Theorem 2.1 (Baccelli et al. 1992): with $A \in \mathbb{R}_{\max }^{n \times n}$ and $B \in \mathbb{R}_{\max }^{n \times m}$, the minimal solution of equation: $X=A \otimes X \oplus B$ is: $X=A^{*} \otimes B$

with $A^{*}=\underset{i \in \mathbb{N}}{\oplus} A^{i}$ being the Kleene star of $A$.

By applying the previous theorem to equations (3), we get to the following explicit form:

$$
X(k)=A \otimes X(k-1) \oplus B \otimes U(k)
$$


with: $A_{0}^{*}=\left(\begin{array}{ll}e & \varepsilon \\ 4 & e\end{array}\right), A=A_{0}^{*} \cdot A_{1}=\left(\begin{array}{ll}\varepsilon & 1 \\ \varepsilon & 5\end{array}\right)$, and $B=A_{0}^{*} \cdot B_{0}=\left(\begin{array}{l}1 \\ 5\end{array}\right)$.

Remark 2.1: Equation (3) and its explicit form (4) feature every TEG (alive nevertheless). It is a standard state representation like the classical representation of linear systems. Thus, it is widely used alike to solve many problems of performance evaluation and control synthesis (Cohen, et al. 1999).

\section{NCTEG modeling in (Max,+) algebra}

\subsection{Definitions and notations}

A net of Conflicting Timed Event Graphs or NCTEG is a set of TEGs noted $\mathcal{G}=\left\{\mathcal{G}_{1}, \mathcal{G}_{2}, \cdots, \mathcal{G}_{N}\right\}$ (e.g. products, jobs, users...) connected to each other by a set of conflict places (e.g. shared resources) noted $\tilde{R}=\left\{\tilde{p}_{1}, \tilde{p}_{2}, \cdots, \tilde{p}_{M}\right\}$. Each timed event graph $\mathcal{G}_{i}$ is connected to a subset of conflict places (or resources) noted $\tilde{R}_{i}$ $\left(\tilde{R}_{i} \subseteq \tilde{R}\right)$. With place $\tilde{p}_{j} \in \tilde{R}_{i}$, we get the generic structure of NCTEG on Fig. 2(a):

$(a)$

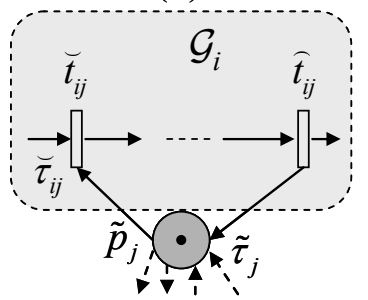

(b)

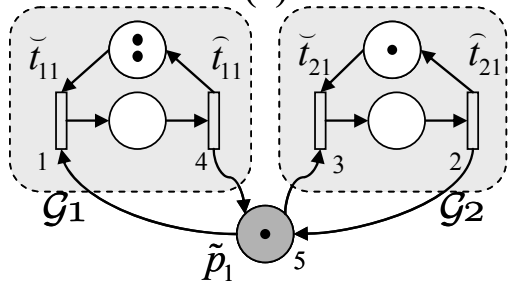

Fig. 2. (a) Generic structure of a NCTEG ( $\tilde{p}_{j} \in \tilde{R}_{i}$ ), (b) example of NCTEG (one conflict place and two TEGs).

For the sake of simplicity, we will adopt the following hypotheses and notations:

- H1) the unique upstream transition of place $\tilde{p}_{j}$ in $\mathcal{G}_{i}$ is noted $\tilde{t}_{i j}$ and the unique downstream transition is noted $\breve{t}_{i j}$ (Fig. 2(a)). We suppose also, without loss of generality (since we can always get to this case by duplicating the transition $\breve{t}_{i j}$ ) that $\breve{t}_{i j}$ has a unique upstream place of conflict $\tilde{p}_{j}$.

- H2) a shared resource is used by at most one user at a time. So, in terms of Petri nets, when the unique token of a place $\tilde{p}_{j}$ is not in this place, only one elementary circuit $\breve{t}_{i j} \cdots \widehat{t}_{i j} \tilde{p}_{j} \breve{t}_{i j}$, among all the others that involve place $\tilde{p}_{j}$, contains it at a time. A resource is indeed either idle or being used by one among the $N$ TEGs.

- H3) each TEG $\mathcal{G}_{i}$ is alive, represented by its state form:

$$
X_{i}\left(k_{i}\right)=A_{0 i} \otimes X_{i}\left(k_{i}\right) \oplus A_{1 i} \otimes X_{i}\left(k_{i}-1\right) \oplus B_{0 i} \otimes U_{i}\left(k_{i}\right)
$$


where: $X_{i}=\left(\begin{array}{llll}x_{i 1} & x_{i 2} & \cdots & x_{i n_{i}}\end{array}\right)^{t}$ is the state vector whose dimension is $n_{i}$ and $U_{i}=\left(\begin{array}{llll}u_{i 1} & u_{i 2} & \cdots & u_{i m_{i}}\end{array}\right)^{t}$ is the input vector whose dimension is $m_{i}$. So, $m_{i}$ is the number of input transitions in TEG $\mathcal{G}_{i}$ and $n_{i}$ the number of the other transitions.

In the remaining of this study, we also adopt the following notations:

- N1) the holding time associated to place $\tilde{p}_{j}$ is denoted $\tilde{\tau}_{j}$ and the firing time of $\breve{t}_{i j}$ is denoted $\breve{\tau}_{i j}$ (see Fig. 2(a)).

- N2) the $i^{\text {th }}$ row of a matrix $A$ is denoted $A(i,:)$ whereas the intersection element of the $i^{\text {th }}$ row and the $j^{\text {th }}$ column is denoted $A(i, j)$.

\subsection{NCTEG modeling in (Max,+) Algebra}

In studying TEGs, daters are usually associated to the transitions. In our approach however, we also associate a dater to the token of every place of conflict $\tilde{p}_{j} \in \tilde{R}$. The date of availability of this token for the $l_{j}^{\text {th }}$ time is denoted $\tilde{\psi}_{j}\left(l_{j}\right)$. Intuitively, this is the date of availability of the shared resource $\tilde{p}_{j}$ after being used $l_{j}$ times.

Let us suppose that every transition of TEG $\mathcal{G}_{i}$ has already fired $\left(k_{i}-1\right)$ times. To fire for the $k_{i}^{\text {th }}$ time, each transition needs all its upstream places to have at least one available token. So, a conflicting transition $\breve{t}_{i j}$ needs the token of its upstream place of conflict $\tilde{p}_{j}$ to be available too. Suppose that the token of this place is attributed effectively to $\mathcal{G}_{i}$ when it is available for the $l_{j}^{\text {th }}$ time. So, to write the dater of transition we need to consider two cases, depending of whether the transition is downstream of a place of conflict or not.

1) If $x_{i j}$ is the dater of vector $X_{i}$ associated to transition of conflict $\breve{t}_{i j}$, then we can write:

$x_{i j}\left(k_{i}\right)=A_{0 i}(j,:) \otimes X_{i}\left(k_{i}\right) \oplus A_{1 i}(j,:) \otimes X_{i}\left(k_{i}-1\right) \oplus B_{0 i}(j,:) \otimes U_{i}\left(k_{i}\right) \oplus \widetilde{\tau}_{i j} \otimes \tilde{\psi}_{j}\left(l_{j}\right)$

Equation (6) is written for every transition downstream a place of conflict belonging to $\tilde{R}_{i}$.

2) If dater $x_{i j}$ is associated to a transition which is not downstream a place of conflict, then its expression is unchanged in (5) since it does not depend directly on $\tilde{\psi}_{j}\left(l_{j}\right)$. So, we have:

$x_{i j}\left(k_{i}\right)=A_{0 i}(j,:) \otimes X_{i}\left(k_{i}\right) \oplus A_{1 i}(j,:) \otimes X_{i}\left(k_{i}-1\right) \oplus B_{0 i}(j,:) \otimes U_{i}\left(k_{i}\right)$

Or indifferently, by adding a null term as follows:

$x_{i j}\left(k_{i}\right)=A_{0 i}(j,:) \otimes X_{i}\left(k_{i}\right) \oplus A_{1 i}(j,:) \otimes X_{i}\left(k_{i}-1\right) \oplus B_{0 i}(j,:) \otimes U_{i}\left(k_{i}\right) \oplus \varepsilon \otimes \tilde{\psi}_{j}\left(l_{j}\right)$

So, by combining (6) and (7), we get to the new state form: 


$$
X_{i}\left(k_{i}\right)=A_{0 i} \otimes X_{i}\left(k_{i}\right) \oplus A_{1 i} \otimes X_{i}\left(k_{i}-1\right) \oplus B_{0 i} \otimes U_{i}\left(k_{i}\right) \oplus \underset{\tilde{p}_{j} \in \tilde{R}_{i}}{F_{0 i j}} \otimes \tilde{\psi}_{j}\left(l_{j}\right)
$$

All the involved matrices and vectors in (8) are exactly the same as in (5). The only new elements are the availability dates $\tilde{\psi}_{j}\left(l_{j}\right)$ defined previously and vectors $F_{0 i j}, \quad 1 \leq i \leq N$ and $1 \leq j \leq M$. We can note that vector $F_{0 i j}$ is of the form $\left(\begin{array}{llllll}\varepsilon & \cdots & \breve{\tau}_{i j} & \cdots & \varepsilon\end{array}\right)^{t}$ i.e. its components are all null (equal to $\varepsilon$ ) except for the $j^{t^{t h}}$ one (equal to $\breve{\tau}_{i j}$ ).

By applying Theorem 2.1 to (8), we finally obtain:

$$
X_{i}\left(k_{i}\right)=A_{i} \otimes X_{i}\left(k_{i}-1\right) \oplus B_{i} \otimes U_{i}\left(k_{i}\right) \oplus\left[\bigoplus_{\tilde{p}_{j} \in \tilde{R}_{i}} F_{i j} \otimes \tilde{\psi}_{j}\left(l_{j}\right)\right]
$$

where: $A_{i}=A_{0}^{*} \otimes A_{1 i}, B_{i}=A_{0}^{*} \otimes B_{0 i}$ and $F_{i j}=A_{0}^{*} \otimes F_{0 i j}$.

On the other hand, since the token of $\tilde{p}_{j}$ is consumed by $\mathcal{G}_{i}$ (via the firing of $\breve{t}_{i j}$ ) when it is available for the $l_{j}^{\text {th }}$ time, then it is available for the $\left(l_{j}+1\right)^{\text {th }}$ time by the firing of transition $\bar{t}_{i j}$ for the $k_{i}^{\text {th }}$ time (recall from hypothesis $H 2$ that circuit $\breve{t}_{i j} \cdots \bar{t}_{i j} \tilde{p}_{j} \breve{t}_{i j}$ is safe). Let $\hat{x}_{i j}$ be the component of $X_{i}$ associated to transition $\widehat{t}_{i j}$. Thus, we can write:

$$
\tilde{\psi}_{j}\left(l_{j}+1\right)=\tilde{\tau}_{j} \otimes \hat{x}_{i j}\left(k_{i}\right)
$$

This equation can be rewritten as:

$$
\tilde{\psi}_{j}\left(l_{j}+1\right)=G_{i j} \otimes X_{i}\left(k_{i}\right)
$$

with $G_{i j}$ is a line vector $\left(\begin{array}{lllll}\varepsilon & \cdots & \tilde{\tau}_{j} & \cdots & \varepsilon\end{array}\right)$ whose all components are null except for one (equal to $\tilde{\tau}_{j}$ ) corresponding to transition $\widehat{t}_{i j}$.

To sum up, if the $l_{j}^{\text {th }}$ token of $\tilde{p}_{j}$ contributes to firing the transitions of TEG $\mathcal{G}_{i}$ for the $k_{i}^{\text {th }}$ time (note that because of resources invariance we have: $l_{j}=\sum_{\tilde{p}_{j} \in \tilde{R}_{i}}\left(k_{i}-1\right)$ ), then the following recurrent $(\mathrm{Max},+)$ equations are verified:

$$
\left\{\begin{array}{l}
X_{i}\left(k_{i}\right)=A_{i} \otimes X_{i}\left(k_{i}-1\right) \oplus B_{i} \otimes U_{i}\left(k_{i}\right) \oplus \bigoplus_{\tilde{p}_{j} \in \tilde{R}_{i}} F_{i j} \otimes \tilde{\psi}_{j}\left(l_{j}\right) \\
\tilde{\psi}_{j}\left(l_{j}+1\right)=G_{i j} \otimes X_{i}\left(k_{i}\right) \quad \text { for all } \tilde{p}_{j} \in \tilde{R}_{i}
\end{array}\right.
$$

As can be seen, equations (12) include a parameter, $l_{j}$ in $\tilde{\psi}_{j}\left(l_{j}\right)$, that defines entirely the policy of resources allocation. Hence, these equations represent all the admissible evolutions of NCTEGs. All the involved elements are systematically calculated according to the method explained before. These equations are important and will be the cornerstone of all the results provided later in this paper. 
Example 3.1: to illustrate the previous method, let us consider the following example (note that if the holding time or firing time is not mentioned, then it is equal to 0$)$ :

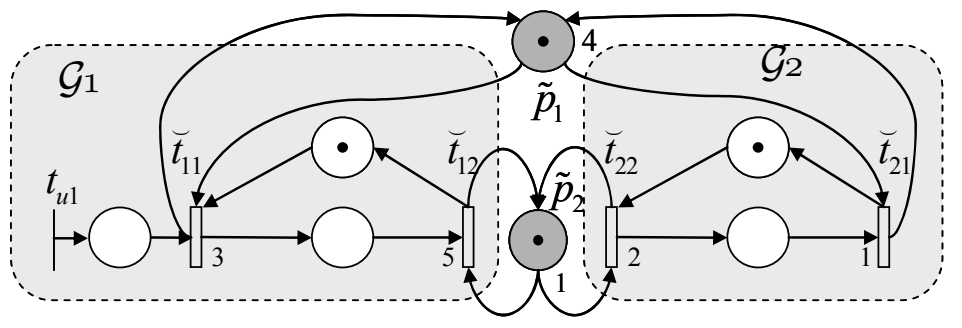

Fig. 3. A NCTEG with two conflict places shared by two TEGs (note that in this example $\widehat{t}_{i j} \equiv \breve{t}_{i j}$ $\forall i, j$, so we use only $\breve{x}$ to refer to the different transitions).

By assigning daters $\breve{x}_{11}, \breve{x}_{12}, \breve{x}_{21}$, and $\breve{x}_{22}$ to transitions $\breve{t}_{11}, \breve{t}_{12}, \breve{t}_{21}$ and $\breve{t}_{22}, \mathcal{G}_{1}$ (without conflicts) is represented by equations:

$\mathcal{G}_{1}:\left\{\begin{array}{l}\breve{x}_{11}\left(k_{1}\right)=3 \otimes u_{1}\left(k_{1}\right) \oplus 3 \otimes \breve{x}_{12}\left(k_{1}-1\right) \\ \breve{x}_{12}\left(k_{1}\right)=5 \otimes \breve{x}_{11}\left(k_{1}\right)\end{array}\right.$

Now, let us take into account the places of conflict. If the token of $\tilde{p}_{1}$ (available for the $l_{1}^{\text {th }}$ time) and the token of $\tilde{p}_{2}$ (available for the $l_{2}^{\text {th }}$ time) are attributed to $\mathcal{G}_{1}$ to enable its transitions to fire for the $k_{1}^{\text {th }}$ time, then we have:

$$
\left\{\begin{array}{l}
\breve{x}_{11}\left(k_{1}\right)=3 \otimes u_{1}\left(k_{1}\right) \oplus 3 \otimes \breve{x}_{12}\left(k_{1}-1\right) \oplus 3 \otimes \tilde{\psi}_{1}\left(l_{1}\right) \\
\breve{x}_{12}\left(k_{1}\right)=5 \otimes \breve{x}_{11}\left(k_{1}\right) \oplus 5 \otimes \tilde{\psi}_{2}\left(l_{2}\right) \\
\tilde{\psi}_{1}\left(l_{1}+1\right)=4 \otimes \breve{x}_{11}\left(k_{1}\right) \\
\tilde{\psi}_{2}\left(l_{2}+1\right)=1 \otimes \breve{x}_{12}\left(k_{1}\right)
\end{array}\right.
$$

These equations are easily brought to form (12):

$$
\left\{\begin{array}{l}
X_{1}(k)=\left(\begin{array}{ll}
\varepsilon & 3 \\
\varepsilon & 8
\end{array}\right) \otimes X_{1}(k-1) \oplus\left(\begin{array}{l}
3 \\
8
\end{array}\right) \otimes U_{1}(k) \oplus\left(\begin{array}{l}
3 \\
8
\end{array}\right) \otimes \tilde{\psi}_{1}\left(l_{1}\right) \oplus\left(\begin{array}{l}
\varepsilon \\
5
\end{array}\right) \otimes \tilde{\psi}_{2}\left(l_{2}\right) \\
\tilde{\psi}_{1}\left(l_{1}+1\right)=\left(\begin{array}{ll}
4 & \varepsilon
\end{array}\right) \otimes X_{1}(k) \\
\tilde{\psi}_{2}\left(l_{2}+1\right)=\left(\begin{array}{ll}
\varepsilon & 1
\end{array}\right) \otimes X_{1}(k)
\end{array}\right.
$$

We let the reader write the equations relative to $\mathcal{G}_{2}$ and get to form (12) with:

$$
\left\{\begin{array}{l}
X_{2}(k)=\left(\begin{array}{ll}
3 & \varepsilon \\
2 & \varepsilon
\end{array}\right) \otimes X_{2}(k-1) \oplus\left(\begin{array}{l}
\varepsilon \\
\varepsilon
\end{array}\right) \otimes U_{2}(k) \oplus\left(\begin{array}{l}
1 \\
\varepsilon
\end{array}\right) \otimes \tilde{\psi}_{1}\left(l_{1}\right) \oplus\left(\begin{array}{l}
3 \\
2
\end{array}\right) \otimes \tilde{\psi}_{2}\left(l_{2}\right) \\
\tilde{\psi}_{1}\left(l_{1}+1\right)=\left(\begin{array}{ll}
4 & \varepsilon
\end{array}\right) \otimes X_{2}(k) \\
\tilde{\psi}_{2}\left(l_{2}+1\right)=\left(\begin{array}{ll}
\varepsilon & 1
\end{array}\right) \otimes X_{2}(k)
\end{array}\right.
$$

Note that all these matrices are constant and independent of resources allocation policy. 


\section{Standard linear $(\mathrm{Max},+)$ equations of NCTEGs}

Let us recall that a linear time-varying system is of the form:

$$
X(k)=A(k-1) \otimes X(k-1) \oplus B(k) \otimes U(k)
$$

This equation would be more suitable than form (12) for most problems resolution. So, one can ask the question: is it possible to bring form (12) to form (15)? Answering this question is indeed crucial since modeling many existing systems using NCTEGs is quite easy (e.g. the TEGs are products and the conflict places are machines) but obtaining directly (15) is not so obvious for some reasons exposed later (Remark 4.2).

To provide an answer to the previous question, one has to know how the shared resources are allocated to the different TEGs (the sequence of allocating the resources). So, if we know which TEG uses a given resource at a given date for a given time, we can deduce which TEG will use it next. So, by using recurrence and equations (12), we can build a time-varying state representation.

\subsection{Definitions}

- D1) In practice, resources are usually allocated according to a sequence (periodic or not). So, we say that a NCTEG evolves according to sequence $\sigma=\mathcal{G}_{j 1} \mathcal{G}_{j 2} \cdots \mathcal{G}_{j(k-1)} \mathcal{G}_{j k} \cdots$, if for each resource represented by $\tilde{p}_{j}$ and shared between two TEGs $\mathcal{G}_{j k}$ and $\mathcal{G}_{j(k+q)}$, the resource $\tilde{p}_{j}$ is first attributed to $\mathcal{G}_{j k}$ and subsequently to $\mathcal{G}_{j(k+q)}$ for all $k \geq 1, q \geq 1$. Then, we say that at the $k^{\text {th }}$ step of sequence $\sigma$ the resources are allocated to $\mathcal{G}_{i}$ if: $\mathcal{G}_{j k}=\mathcal{G}_{i}$.

Example 4.1: $\quad \tilde{R}=\left\{\tilde{p}_{1}, \tilde{p}_{2}, \tilde{p}_{3}\right\}, \quad \tilde{R}_{1}=\left\{\tilde{p}_{1}, \tilde{p}_{3}\right\}, \quad \tilde{R}_{2}=\left\{\tilde{p}_{2}, \tilde{p}_{3}\right\}, \quad \tilde{R}_{3}=\left\{\tilde{p}_{1}, \tilde{p}_{2}\right\}$, $\sigma=\mathcal{G}_{1} \mathcal{G}_{2} \mathcal{G}_{2} \mathcal{G}_{1} \mathcal{G}_{3} \mathcal{G}_{3} \mathcal{G}_{3} \mathcal{G}_{2} \mathcal{G}_{1}$. According to this sequence, the token of $\tilde{p}_{1} \in\left(\tilde{R}_{1} \cap \tilde{R}_{3}\right)$ is to be attributed twice to $\mathcal{G}_{1}$ then 3 times to $\mathcal{G}_{3}$ and finally one time to $\mathcal{G}_{1}$.

\section{Remark 4.1:}

The hypothesis that a whole NCTEG evolves according to an arbitrary sequence, common to all the resources as defined before, does mean that all the admissible evolutions of the NCTEG are swept. The general case is when each shared resource evolves according to its own sequence.

- D2) Let us define the set of indices of TEGs: $S=\{1,2, \cdots, N\}$ and the function: Fre: $\tilde{R} \times S \times \mathbb{N} \mapsto S$ where Fre $\left(\tilde{p}_{j}, i, k_{i}\right)$ provides the index of the TEG that frees resource $\tilde{p}_{j}$ to be used by $\mathcal{G}_{i}$ for the $k_{i}^{\text {th }}$ time.

Example 4.1 (continued): Let us return to example 4.1 and build the following table to explain this function (a cross in a box of the row of $\tilde{p}_{j}$ and column of $\mathcal{G}_{i}$ means $\tilde{p}_{j} \in \tilde{R}_{i}$ ): 


\begin{tabular}{|c|c|c|c|c|c|c|c|c|c|}
\hline$\sigma=$ & $\mathcal{G}_{1}$ & $\mathcal{G}_{2}$ & $\mathcal{G}_{2}$ & $\mathcal{G}_{1}$ & $\mathcal{G}_{3}$ & $\mathcal{G}_{3}$ & $\mathcal{G}_{3}$ & $\mathcal{G}_{2}$ & $\mathcal{G}_{l}$ \\
\hline$\tilde{p}_{1}$ & $\mathrm{X}$ & & & $\rightarrow \mathrm{x}$ & $\mathrm{X}$ & $\mathrm{X}$ & $\mathrm{x}$ & & $\mathrm{x}$ \\
\hline$\tilde{p}_{2}$ & & $\mathrm{x}$ & $\mathrm{x}$ & & $\mathrm{X}$ & $\mathrm{x}$ & & $\rightarrow \mathrm{x}$ & \\
\hline$\tilde{p}_{3}$ & $\mathrm{X}$ & $x-$ & $\rightarrow \mathrm{x}$ & $\mathrm{x}$ & & & & $\rightarrow \mathrm{x}$ & $\mathrm{x}$ \\
\hline
\end{tabular}

The arrows in the table simplify the following samples of function Fre: $\operatorname{Fre}\left(\tilde{p}_{1}, 1,2\right)=1, \operatorname{Fre}\left(\tilde{p}_{2}, 2,3\right)=3, \operatorname{Fre}\left(\tilde{p}_{3}, 2,2\right)=2$, and $\operatorname{Fre}\left(\tilde{p}_{3}, 2,3\right)=1$. For instance Fre $\left(\tilde{p}_{3}, 2,3\right)=1$ means that $\mathcal{G}_{1}$ frees $\tilde{p}_{3}$ to be used by $\mathcal{G}_{2}$ for the $3^{\text {rd }}$ time.

Theorem 4.1: A NCTEG verifying the hypotheses of Section 3.1 and evolving according to an arbitrary sequence $\sigma=\mathcal{G}_{j 1} \mathcal{G}_{j 2} \cdots \mathcal{G}_{j(k-1)} \mathcal{G}_{j k} \cdots$, has an equivalent Linear Time-Varying $($ Max, + ) representation.

Proof: Let us suppose that a NCTEG verifies the hypotheses of Section 3.1 and evolves according to a sequence $\sigma$. So, we can write, from $1^{\text {st }}$ equation in (12), the following form:

$$
X_{i}\left(k_{i}\right)=A_{i} \otimes X_{i}\left(k_{i}-1\right) \oplus B_{i} \otimes U_{i}\left(k_{i}\right) \oplus \underset{\tilde{p}_{j} \in \tilde{R}_{i}}{F_{i j}} \otimes \tilde{\psi}_{j}\left(l_{j}\right)
$$

Suppose that TEG $\mathcal{G}_{i}$ frees resource $\tilde{p}_{j}$ to be used by $\mathcal{G}_{i}$ for the $k_{i}^{\text {th }}$ time. So, by using the $2^{\text {nd }}$ equation in (12) we can write:

$\tilde{\psi}_{j}\left(l_{j}\right)=G_{i^{\prime} j} \otimes X_{i^{\prime}}\left(k_{i^{\prime}}-1\right)$

Since by definition $i^{\prime}=\operatorname{Fre}\left(\tilde{p}_{j}, i, k_{i}\right)$, the previous expression becomes:

$$
\tilde{\psi}_{j}\left(l_{j}\right)=G_{\operatorname{Fre}\left(\tilde{p}_{j}, i, k_{i}\right) j} \otimes X_{\operatorname{Fre}\left(\tilde{p}_{j}, i, k_{i}\right)}\left(k_{\operatorname{Fre}\left(\tilde{p}_{j}, i, k_{i}\right)}-1\right)
$$

So, by replacing (17) in (16), we obtain:

$$
X_{i}\left(k_{i}\right)=A_{i} \otimes X_{i}\left(k_{i}-1\right) \oplus B_{i} \otimes U_{i}\left(k_{i}\right) \oplus\left[\bigoplus_{\tilde{p}_{j} \in \tilde{R}_{i}} F_{i j} \otimes G_{F r e\left(\tilde{p}_{j}, i, k_{i}\right) j} \otimes X_{F r e\left(\tilde{p}_{j}, i, k_{i}\right)}\left(k_{F r e\left(\tilde{p}_{j}, i, k_{i}\right)}-1\right)\right]
$$

Before going any further, let us explain how to handle vectors and matrices whose entries are vectors and matrices respectively.

Example 4.2: $a=\left(\begin{array}{ll}1 & 3 \\ e & \varepsilon\end{array}\right), b=\left(\begin{array}{l}1 \\ 1\end{array}\right), c=\left(\begin{array}{ll}\varepsilon & \varepsilon\end{array}\right)$, and $d=(5)$.

A matrix of matrices is for instance: $\bar{A}=\left(\begin{array}{ll}a & b \\ c & d\end{array}\right)$ and a vector of vectors is $\bar{B}=\left(\begin{array}{l}b \\ d\end{array}\right)$.

So, $\bar{A}(1,1)=a, \bar{A}(1,2)=b, \bar{A}(2,1)=c, \bar{A}(2,2)=d \bar{B}(1)=b$, and $\bar{B}(2)=d$. 
Let us then define the vector of vectors $\bar{X}(k)$ (resp. $\bar{U}(k))$ relative to the $(k-1)^{\text {th }}$ step of sequence $\sigma$ as follows:

$\bar{X}(k-1)=\left[\begin{array}{llllll}X_{1}\left(k_{1}-1\right) & X_{2}\left(k_{2}-1\right) & \cdots & X_{i}\left(k_{i}-1\right) & \cdots & X_{N}\left(k_{N}-1\right)\end{array}\right]^{t}$,

$\bar{U}(k-1)=\left[\begin{array}{llllll}U_{1}\left(k_{1}-1\right) & U_{2}\left(k_{2}-1\right) & \cdots & U_{i}\left(k_{i}-1\right) & \cdots & U_{N}\left(k_{N}-1\right)\end{array}\right]^{t}$,

where $\left(k_{i}-1\right)$ is the number of occurrences of TEG $\mathcal{G}_{i}$ at the $(k-1)^{\text {th }}$ step of $\sigma$. It

follows that: $k-1=\sum_{i=1}^{N}\left(k_{i}-1\right)$.

If the $k^{\text {th }}$ step of $\sigma$ corresponds to TEG $\mathcal{G}_{i}$, then we also (according to the previous definition of vector $\bar{X}$ ) write:

$\bar{X}(k)=\left(\begin{array}{llllll}X_{1}\left(k_{1}-1\right) & X_{2}\left(k_{2}-1\right) & \cdots & X_{i}\left(k_{i}\right) & \cdots & \left.X_{N}\left(k_{N}-1\right)\right)^{t}\end{array}\right.$

The question is then, how to write $\bar{X}(k)$ as a function of $\bar{X}(k-1)$ ?

We will write each line of $\bar{X}(k)$ as function of $\bar{X}(k-1)$ and then deduce the relation between the whole vector $\bar{X}(k)$ and $\bar{X}(k-1)$.

The first line $\bar{X}(k)(1)$ of $\bar{X}(k)$ is equal to $X_{1}\left(k_{1}-1\right)$ and the first line of $\bar{X}(k-1)$ is equal to $X_{1}\left(k_{1}-1\right)$ too. So, we can simply write:

$X_{1}\left(k_{1}-1\right)=X_{1}\left(k_{1}-1\right)$

Or by using $\bar{X}(k)$ and $\bar{X}(k-1)$ as:

$\bar{X}(k)(1)=\left(\begin{array}{llll}I d_{n_{1}} & \bar{\varepsilon}_{n_{1} n_{2}} & \cdots & \bar{\varepsilon}_{n_{1} n_{N}}\end{array}\right) \otimes \bar{X}(k-1)$,

where $I d_{n_{1}}$ and $\bar{\varepsilon}_{n_{1} n_{i}}$ are respectively $n_{1} \times n_{1}$ identity matrix and $n_{1} \times n_{i}$ null matrix.

We can repeat the same procedure for the second line of $\bar{X}(k)$ to obtain:

$\bar{X}(k)(2)=\left(\begin{array}{llll}\bar{\varepsilon}_{n_{2} n_{1}} & I d_{n_{2}} & \cdots & \bar{\varepsilon}_{n_{2} n_{N}}\end{array}\right) \otimes \bar{X}(k-1)$

So, we repeat the same procedure for every line $p \neq i$ of $\bar{X}(k)$ and obtain:

$\bar{X}(k)(p)=\left(\begin{array}{lllllll}\bar{\varepsilon}_{n_{p} n_{1}} & \bar{\varepsilon}_{n_{p} n_{2}} & \cdots & I d_{n_{p}} & \cdots & \bar{\varepsilon}_{n_{p} n_{(N-1)}} & \bar{\varepsilon}_{n_{p} n_{N}}\end{array}\right) \otimes \bar{X}(k-1)$

In case of line $p=i$ however, we rather use equation (18):

$\bar{X}(k)(i)=X_{i}\left(k_{i}\right)=A_{i} \otimes X_{i}\left(k_{i}-1\right) \oplus B_{i} \otimes U_{i}\left(k_{i}\right) \oplus\left[\bigoplus_{\tilde{p}_{j} \in \tilde{R}_{i}} F_{i j} \otimes G_{\operatorname{Fre}\left(\tilde{p}_{j}, i, k_{i}\right) j} \otimes X_{\operatorname{Fre}\left(\tilde{p}_{j}, i, k_{i}\right)}\left(k_{F r e\left(\tilde{p}_{j}, i, k_{i}\right)}-1\right)\right]$

By isolating the term multiplied by $X_{i}\left(k_{i}-1\right)$ we get to: 


$$
\begin{aligned}
& \bar{X}(k)(i)=\left[A_{i} \oplus\left(\bigoplus_{\operatorname{Fre}\left(\tilde{p}_{j}, i, k_{i}\right)=i} F_{i j} \otimes G_{i j}\right)\right] \otimes X_{i}\left(k_{i}-1\right) \oplus B_{i} \otimes U_{i}\left(k_{i}\right) \\
& \oplus\left[\underset{\tilde{p}_{j} \in \tilde{R}_{i} / F r e\left(\tilde{p}_{j}, i, k_{i}\right) \neq i}{F_{i j}} \otimes G_{\operatorname{Fre}\left(\tilde{p}_{j}, i, k_{i}\right) j} \otimes X_{\operatorname{Fre}\left(\tilde{p}_{j}, i, k_{i}\right)}\left(k_{\operatorname{Fre}\left(\tilde{p}_{j}, i, k_{i}\right)}-1\right)\right]
\end{aligned}
$$

This equation is of the form:

$$
\bar{X}(k)(i)=\alpha_{i} \otimes X_{i}\left(k_{i}-1\right) \oplus B_{i} \otimes U_{i}\left(k_{i}\right) \oplus\left[\bigoplus_{q \neq i} \beta_{q} \otimes X_{q}\left(k_{q}-1\right)\right]
$$

It can be rewritten using $\bar{X}(k-1)$ as:

$$
\bar{X}(k)(i)=\alpha_{i} \otimes \bar{X}(k-1)(i) \oplus\left[\bigoplus_{q \neq i} \beta_{q} \otimes \bar{X}(k-1)(q)\right] \oplus B_{i} \otimes U_{i}\left(k_{i}\right)
$$

Or:

$$
\bar{X}(k)(i)=\left(\begin{array}{lllll}
\beta_{1} & \cdots & \alpha_{i} & \cdots & \beta_{N}
\end{array}\right) \otimes \bar{X}(k-1)(i) \oplus B_{i} \otimes U_{i}\left(k_{i}\right)
$$

Where: $\alpha_{i}=A_{i} \oplus\left(\underset{\operatorname{Fre}\left(\tilde{p}_{j}, i, k_{i}\right)=i}{F_{i j}} F_{i j}\right)$ and $\beta_{q}=F_{i j} \otimes G_{\operatorname{Fre}\left(\tilde{p}_{j}, i, k_{i}\right) j}$

To sum up, we have the following equations:

$$
\begin{aligned}
& \bar{X}(k)(1)=\left(\begin{array}{llll}
I d_{n_{1}} & \bar{\varepsilon}_{n_{2}} & \cdots & \bar{\varepsilon}_{n_{N}}
\end{array}\right) \otimes \bar{X}(k-1) \\
& \bar{X}(k)(2)=\left(\begin{array}{llll}
\bar{\varepsilon}_{n_{1}} & I d_{n_{2}} & \cdots & \bar{\varepsilon}_{n_{i}}
\end{array}\right) \otimes \bar{X}(k-1) \\
& \bar{X}(k)(i)=\left(\begin{array}{lllll}
\beta_{1} & \cdots & \alpha_{i} & \cdots & \beta_{N}
\end{array}\right) \otimes \bar{X}(k-1)(i) \oplus B_{i} \otimes \bar{U}(k)(i) \\
& \bar{X}(k)(N)=\left(\begin{array}{llll}
\bar{\varepsilon}_{n_{1}} & \cdots & \bar{\varepsilon}_{n_{(N-1)}} & I d_{n_{N}}
\end{array}\right) \otimes \bar{X}(k-1)
\end{aligned}
$$

These equations are equivalent to:

$$
\begin{aligned}
\bar{X}(k) & =\left(\begin{array}{cccccc}
I d_{n_{1}} & \bar{\varepsilon}_{n_{1} n_{2}} & \cdots & & \bar{\varepsilon}_{n_{1} n_{N}} \\
\bar{\varepsilon}_{n_{2} n_{1}} & I d_{n_{2}} & \bar{\varepsilon}_{n_{2} n_{3}} & \cdots & \bar{\varepsilon}_{n_{2} n_{N}} \\
\vdots & & \ddots & \ddots & \vdots \\
\beta_{1} & \cdots & \alpha_{i} & \cdots & \beta_{N} \\
\vdots & & & & \vdots \\
\bar{\varepsilon}_{n_{N} n_{1}} & \bar{\varepsilon}_{n_{N} n_{2}} & \cdots & & \bar{\varepsilon}_{n_{N} n_{(N-1)}} & I d_{n_{N}}
\end{array}\right) \otimes \bar{X}(k-1) \\
& \oplus\left(\begin{array}{ccccc}
\bar{\varepsilon}_{n_{1} m_{1}} & \bar{\varepsilon}_{n_{1} m_{2}} & & \cdots & \bar{\varepsilon}_{n_{1} m_{M}} \\
\vdots & & \ddots & \ddots & \vdots \\
\bar{\varepsilon}_{n_{i} m_{1}} & \cdots & B_{i} & \cdots & \bar{\varepsilon}_{n_{i} m_{M}} \\
\vdots & & & & \vdots \\
\bar{\varepsilon}_{n_{N} m_{1}} & \bar{\varepsilon}_{n_{N} m_{2}} & \cdots & & \bar{\varepsilon}_{n_{N} m_{M}}
\end{array}\right) \otimes \bar{U}(k)
\end{aligned}
$$

This is obviously a linear time-varying $(\mathrm{Max},+)$ system of the form:

$$
\bar{X}(k)=\bar{A}(k-1) \otimes \bar{X}(k-1) \oplus \bar{B}(k) \otimes \bar{U}(k),
$$


where matrices of matrices $\bar{A}(k-1)$ and $\bar{B}(k)$ are defined as:

$\bar{A}(k-1)(p, q)= \begin{cases}I_{n_{p}} & \text { if }(p=q \text { and } p \neq i) \\ \alpha_{i} \quad \text { if }(p=q=i) \\ \beta_{q} \quad \text { if }(p=i \text { and } q \neq i) \\ \bar{\varepsilon}_{n_{p} n_{q}} \text { otherwise }\end{cases}$

$\bar{B}(k)(p, q)= \begin{cases}B_{i} & \text { if }(p=q=i) \\ \bar{\varepsilon}_{n_{p} m_{q}} & \text { otherwise }\end{cases}$

Finally, the sought linear representation is obtained and this concludes the proof of Theorem 4.1.

Note that $\bar{A}(k-1)(p, q)$ and $\bar{B}(k)(p, q)$ are not scalars but matrices. They are multiplied by the elements of $\bar{X}$ and $\bar{U}$ which are vectors.

\section{Remarks 4.2:}

- It could be thought that since we can represent a system using a NCTEG and subsequently equations (19), then why not do it from the beginning without passing through the NCTEG modeling? Indeed, equations (19) might represent a TEG (with time-varying parameters nevertheless), equivalent to the NCTEG. However, if we look closely at matrix $\bar{A}(k)$, we will notice that some of its components are, over time, sometimes null (equals $\bar{\varepsilon}$ ) and sometimes not. This means literally that some places of the TEG sometimes exist and sometimes disappear. This is obviously not a usual feature with regard to Petri nets and therefore not a natural modeling of systems (see Example 6.1).

- As a straightforward result from the previous theorem, a NCTEG, verifying the conditions of Section 3.1 (especially the TEGs to be alive) and evolving according to an arbitrary sequence, is necessarily alive. Indeed, since the NCTEG is initially alive, the recurrent linear time-varying equations are written according to the previous theorem. The evolution of the NCTEG can be calculated until any horizon $k$. So, the firing of all the transitions for the $k^{\text {th }}$ time $(k \in \mathbb{N})$ is possible and the date of each firing is finite. This is however not always true, even if the TEGs are alive, if each resource is allocated according to its own sequence (general case). The deadlock in this case would be caused by the non feasibility of the adopted scheduling for instance.

\section{5. $($ Max, + ) equations of cyclic NCTEGs}

Equation (19) can be obtained whatever is the NCTEG evolution sequence. In practice nonetheless, many systems are repetitive and evolve according to a periodic sequence of the form $\sigma=\sigma_{0} \sigma_{0} \sigma_{0} \ldots$ where $\sigma_{0}$ is the basic sequence to be repeated. Let us suppose: $\sigma_{0}=\mathcal{G}_{j 1} \mathcal{G}_{j 2} \cdots \mathcal{G}_{j(T-1)} \mathcal{G}_{j T}$. This means that $\sigma$ is $T$-periodic It can be easily checked that given this periodic pattern, we have:

$\operatorname{Fre}\left(\tilde{p}_{j}, i, k_{i}+T\right)=\operatorname{Fre}\left(\tilde{p}_{j}, i, k_{i}\right)$ for every triplet $\left(\tilde{p}_{j}, i, k_{i}\right)$. It follows that matrix $\bar{A}$ and $\bar{B}$ are $T$-periodic i.e. $\bar{A}(k+T)=\bar{A}(k)$ and $\bar{B}(k+T)=\bar{B}(k)$. 
Hence, we can apply a well known result in conventional theory related to the classical periodic linear systems (Bolzern et al. 1986), (Misra, 1996) and similarly used in $(\mathrm{Max},+)$ algebra (Lahaye et al. 2004). This result is a transformation of a periodic time-varying linear system into an time-invariant one using the notion of monodromy.

Let us define the transition matrix relative to matrix $\bar{A}$ as:

$\Phi(i, i-q)=\left\{\begin{array}{l}\bar{A}(i-1) \otimes \bar{A}(i-2) \otimes \cdots \bar{A}(i-q) \text { if } q \geq 1 . \\ I_{d} \quad \text { if } q=0\end{array}\right.$.

Note that $\Phi$ is $T$-periodic i.e. $\Phi(i+T, i+T-q)=\Phi(i, i-q)$.

Using (19), it follows that:

$\bar{X}(2)=\bar{A}(1) \otimes \bar{X}(1) \oplus \bar{B}(2) \otimes \bar{U}(2)$

$\bar{X}(3)=\bar{A}(2) \otimes \bar{X}(2) \oplus \bar{B}(3) \otimes \bar{U}(3)$

$=\Phi(3,1) \otimes \bar{X}(1) \oplus \Phi(3,2) \otimes \bar{B}(2) \otimes \bar{U}(2) \oplus \Phi(3,3) \otimes \bar{B}(3) \otimes \bar{U}(3)$

By recurrence, we get to:

$$
\begin{aligned}
& \bar{X}(1+T)=\Phi(1+T, 1) \otimes \bar{X}(1) \oplus \\
& \bigoplus_{q=1}^{T} \Phi(1+T, 1+q) \otimes \bar{B}(1+q) \otimes \bar{U}(1+q)
\end{aligned}
$$

By adding $(l-1) \cdot T$ in vectors indices of (23) and using the periodicity of $\Phi$, we get to:

$$
\begin{aligned}
& \bar{X}(1+l \cdot T)=\Phi(1+T, 1) \otimes \bar{X}(1+(l-1) \cdot T) \oplus \\
& \bigoplus_{q=1}^{T} \Phi(1+T, 1+q) \otimes \bar{B}(1+q) \otimes \bar{U}(1+(l-1) \cdot T+q)
\end{aligned}
$$

Let us set $\overline{\bar{X}}(l)=\bar{X}(1+l \cdot T)$ and $\overline{\bar{U}}(l)=(\bar{U}(1+(l-1) \cdot T+1) \cdots \bar{U}(1+(l-1) \cdot T+T))^{t}$. Equation (24) is therefore a standard time-invariant system:

$$
\overline{\bar{X}}(l)=\overline{\bar{A}} \otimes \overline{\bar{X}}(l-1) \oplus \overline{\bar{B}} \otimes \overline{\bar{U}}(l)
$$

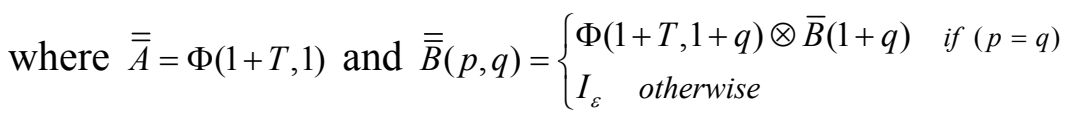

Note that we could start from any initial step $k_{0}$ in (22). In this case, we find matrix $\overline{\bar{A}}=\Phi\left(k_{0}+T, k_{0}\right)$. The state matrix $\overline{\bar{A}}$ is then called monodromy matrix at $k_{0}$.

\section{Application: modeling and performance evaluation of jobshops}

Different applications of the NCTEG-based method presented previously can be considered: performance evaluation, control synthesis, scheduling optimization, 
resources use optimization, event-driven simulation, etc. As an example of application, we present some performances evaluation of the well-known systems called jobshops.

Definition 6.1: as defined for instance in Gaubert et al. (1999), a jobshop is specified by:

- A finite set $\mathcal{R}=\left\{r_{1}, r_{2}, \cdots, r_{M}\right\}$ of resources (machines)

- A finite set $\mathcal{T}$ of elementary tasks

- Each task $a \in \mathcal{T}$ is to be executed on a single machine $R(a) \in \mathcal{R}$ (hypothesis) during a duration $\tau(a)$.

- A finite set of jobs $\mathcal{J}$. Each job $J=a_{1} a_{2} \cdots a_{m} \in \mathcal{J}$ is composed of a sequence of the elementary tasks, each task $a$ belonging to a unique job $J(a)$ (hypothesis). We say that a unit of job $J$ is produced every time the sequence $J$ is executed.

According to this definition and the considered hypotheses, one can draw the generic NCTEG model of every job $J$ as in Fig. 4, duration $\tau_{i}$ being equal to $\tau\left(a_{i}\right)$. For clarity reasons, we omitted to represent the other transitions from/to the conflict places.

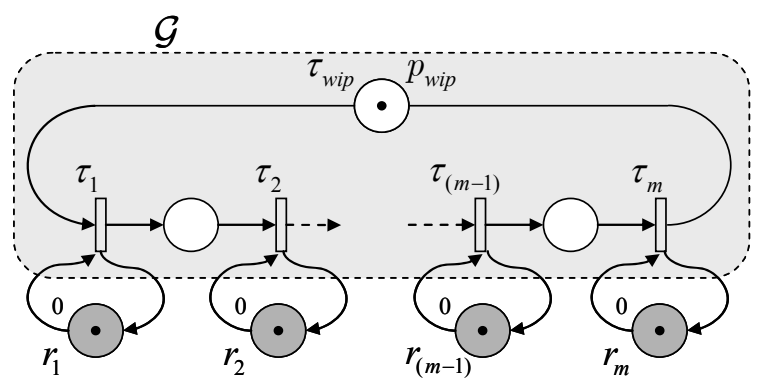

Fig. 4. A NCTEG-based model of a jobshop.

Note that graph $\mathcal{G}$, representing job $J$, involves a place noted $p_{\text {wip }}$ whose delay is $\tau_{w i p}$. This place represents the number of works in process (WIP) i.e. the number of units of job $J$ that are allowed to be processed in parallel. The number of tokens of place $p_{\text {wip }}$ defines the WIP (this may represent for instance the number of pallets that are allocated to a given part type in a manufacturing system). In Fig. 4, there is only one token in $p_{\text {wip }}$. So, this job $J$ is said to be safe. Thus, the processing of a task of $J$ cannot start again while all the tasks of the current cycle are not finished. If all the jobs of a jobshop are safe, the jobshop is safe.

The matrices of the $(\mathrm{Max},+)$ representation of the NCTEG-based model are easily calculated. Let us write the $(\mathrm{Max},+)$ equations of graph $\mathcal{G}$ in Fig. 4 :

$$
\left\{\begin{array}{l}
x_{1}(k)=\tau_{1} \otimes\left[\tau_{\text {wip }} \otimes x_{m}(k-1) \oplus \psi_{1}\left(l_{1}\right)\right] \\
x_{2}(k)=\tau_{2} \otimes\left[x_{1}(k) \oplus \psi_{2}\left(l_{2}\right)\right] \\
\vdots \\
x_{m}(k)=\tau_{m} \otimes\left[x_{(m-1)}(k) \oplus \psi_{m}\left(l_{m}\right)\right]
\end{array}\right.
$$


We have also:

$$
\left\{\tilde{\psi}_{j}\left(l_{j}+1\right)=e \otimes x_{j}(k) \quad \text { for } j=1, \ldots, m\right.
$$

These equations can be brought to a state representation:

$$
\left\{\begin{array}{l}
X\left(k_{i}\right)=A_{i} \otimes X_{i}\left(k_{i}-1\right) \oplus \bigoplus_{j=1}^{m} F_{i j} \otimes \tilde{\psi}_{j}\left(l_{j}\right) \\
\tilde{\psi}_{j}\left(l_{j}+1\right)=G_{i j} \otimes X_{i}\left(k_{i}\right) \quad \text { for } j=1, \ldots, m
\end{array}\right.
$$

where $A_{i}=\tau_{\text {wip }} \otimes\left(\begin{array}{cccc}\varepsilon & \cdots & \varepsilon & \tau_{1} \\ \varepsilon & \cdots & \varepsilon & \tau_{2} \otimes \tau_{1} \\ \vdots & & \vdots & \vdots \\ \varepsilon & \cdots & \varepsilon & \tau_{m} \otimes \cdots \tau_{2} \otimes \tau_{1}\end{array}\right), \quad F_{i 1}=\left(\begin{array}{c}\tau_{1} \\ \tau_{2} \otimes \tau_{1} \\ \vdots \\ \tau_{m} \otimes \cdots \tau_{2} \otimes \tau_{1}\end{array}\right), \quad F_{i 2}=\left(\begin{array}{c}\varepsilon \\ \tau_{2} \\ \tau_{3} \otimes \tau_{2} \\ \vdots \\ \tau_{m} \otimes \tau_{m-1} \cdots \otimes \tau_{2}\end{array}\right)$,

$\ldots, F_{i m}=\left(\begin{array}{c}\varepsilon \\ \varepsilon \\ \varepsilon \\ \vdots \\ \tau_{m}\end{array}\right), G_{i 1}=\left(\begin{array}{llll}e & \varepsilon & \cdots & \varepsilon\end{array}\right), G_{i 2}=\left(\begin{array}{llll}\varepsilon & e & \cdots & \varepsilon\end{array}\right), \ldots, G_{i m}=\left(\begin{array}{llll}\varepsilon & \varepsilon & \cdots & e\end{array}\right)$.

Hence, the matrices relative to each job (graph) are calculated systematically, without passing through the $(\mathrm{Max},+)$ equations.

\section{Remark 6.1}

Contrary to Gaubert et al. (1999), where only safe jobshops were studied using the heaps-of-pieces approach, we can consider, using our method, any number of tokens in $p_{\text {wip }}$. Such a condition has indeed not been posed in our hypotheses (our hypothesis of boundedness is related only to elementary circuits $\breve{t}_{i j} \cdots \bar{t}_{i j} \tilde{p}_{j} \breve{t}_{i j}$ of the NCTEG not the whole of it). All we have to do is transform this place $p_{\text {wip }}$ into a series of places containing one token to obtain the first order $(\mathrm{Max},+)$ representations of the graphs (Baccelli et al. 1992). The delays associated to the added places and transitions are all zeros, except for one that equals the delay of place $p_{\text {wip }}$. In case of three tokens for instance, the transformation is depicted in Fig. 5.

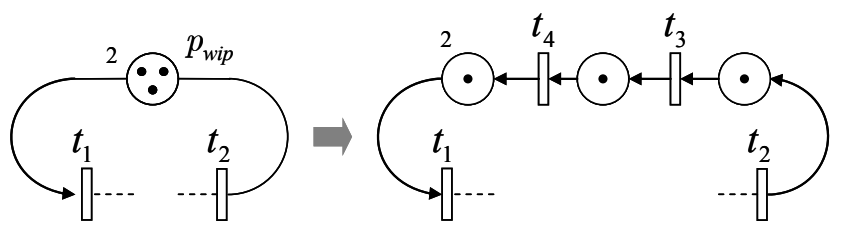

Fig. 5. Example of a NCTEG transformation in case of a 3-bounded jobshop.

We add two transitions $t_{3}$ and $t_{4}$. The $3^{\text {rd }}$ order equations of this TEG can then be rewritten in an equivalent first order form as follows: 
$x_{1}(k)=2 \otimes x_{2}(k-3) \Rightarrow\left\{\begin{array}{l}x_{1}(k)=2 \otimes x_{4}(k-1) \\ x_{3}(k)=e \otimes x_{2}(k-1) \\ x_{4}(k)=e \otimes x_{3}(k-1)\end{array}\right.$

Example 6.1: To illustrate how to use systematically the results above, we consider an example studied in Hillion et al. (1989) and Gaubert et al. (1999). It is a manufacturing system constituted of three machines $r_{1}, r_{2}$ and $r_{3}$ that produce three types of parts represented by TEGs $\mathcal{G}_{1}, \mathcal{G}_{2}$ and $\mathcal{G}_{3}$ (Fig. 6). Note that: $\tilde{R}_{1}=\left\{r_{1}, r_{2}, r_{3}\right\}, \tilde{R}_{2}=\left\{r_{2}, r_{3}\right\}, \tilde{R}_{3}=\left\{r_{1}, r_{3}\right\}$.

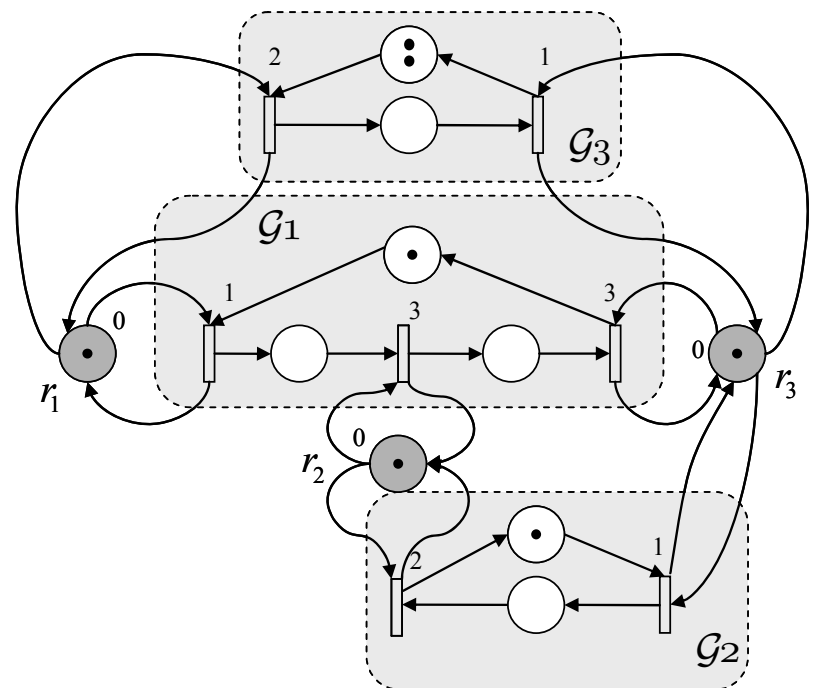

Fig. 6. A NCTEG model of a manufacturing system (jobshop) involving three machines producing three types of parts.

Let us associate availability dates $\tilde{\psi}_{1}, \tilde{\psi}_{2}, \tilde{\psi}_{3}$ to the tokens of places $r_{1}, r_{2}$ and $r_{3}$.

So, according to (28) the matrices relative to TEG $\mathcal{G}_{1}$ are directly calculated as:

$$
\begin{aligned}
& A_{1}=\left(\begin{array}{ccc}
\varepsilon & \varepsilon & 1 \\
\varepsilon & \varepsilon & 1+3 \\
\varepsilon & \varepsilon & 1+3+3
\end{array}\right), F_{11}=\left(\begin{array}{c}
1 \\
1+3 \\
1+3+3
\end{array}\right), F_{12}=\left(\begin{array}{c}
\varepsilon \\
3 \\
3+3
\end{array}\right) \text { and } F_{13}=\left(\begin{array}{l}
\varepsilon \\
\varepsilon \\
3
\end{array}\right) . \\
& G_{11}=\left(\begin{array}{lll}
e & \varepsilon & \varepsilon
\end{array}\right), G_{12}=\left(\begin{array}{lll}
\varepsilon & e & \varepsilon
\end{array}\right), \text { and } G_{13}=\left(\begin{array}{lll}
\varepsilon & \varepsilon & e
\end{array}\right) .
\end{aligned}
$$

The matrices relative to $\mathcal{G}_{2}$ are:

$$
A_{2}=\left(\begin{array}{ll}
\varepsilon & 1 \\
\varepsilon & 3
\end{array}\right), F_{23}=\left(\begin{array}{c}
1 \\
1+2
\end{array}\right), F_{22}=\left(\begin{array}{l}
\varepsilon \\
2
\end{array}\right), G_{23}=\left(\begin{array}{ll}
e & \varepsilon
\end{array}\right) \text {, and } G_{22}=\left(\begin{array}{ll}
\varepsilon & e
\end{array}\right) .
$$

We let the reader make the transformation (as on Fig. 5) of the place with two tokens in TEG $\mathcal{G}_{3}$. The resulting matrices are: 
$A_{3}=\left(\begin{array}{lll}\varepsilon & \varepsilon & 2 \\ \varepsilon & \varepsilon & 3 \\ \varepsilon & e & \varepsilon\end{array}\right), F_{31}=\left(\begin{array}{l}2 \\ 3 \\ \varepsilon\end{array}\right), F_{33}=\left(\begin{array}{l}\varepsilon \\ 1 \\ \varepsilon\end{array}\right), G_{31}=\left(\begin{array}{lll}e & \varepsilon & \varepsilon\end{array}\right)$ and $G_{33}=\left(\begin{array}{lll}\varepsilon & e & \varepsilon\end{array}\right)$.

All these matrices are calculated once and for all, whatever is the considered sequence of the jobshop evolution.

- Production rate evaluation: as a first application, we will consider a periodic case and calculate the asymptotic production rate of the jobshop. Let us consider for instance the basic sequence: $\sigma_{0}=\mathcal{G}_{1} \mathcal{G}_{2} \mathcal{G}_{3} \mathcal{G}_{3}$. We first calculate Fre $\left(\tilde{p}_{j}, i, k_{i}\right)$ relative to the three resources:

Table 2: function Fre values in case of Example 6.1

\begin{tabular}{|c|c|c|c|c|c|}
\hline & $\sigma_{0}=$ & $\mathcal{G}_{1}$ & $\mathcal{G}_{2}$ & $\mathcal{G}_{3}$ & $\mathcal{G}_{3}$ \\
\hline $\begin{array}{l}\operatorname{Fre}\left(\tilde{p}_{1}, 1,1\right)=3, \operatorname{Fre}\left(\tilde{p}_{1}, 3,1\right)=1, \\
\operatorname{Fre}\left(\tilde{p}_{1}, 3,2\right)=3\end{array}$ & $\tilde{p}_{1}$ & ${ }^{x}-$ & & $\rightarrow x-$ & $\rightarrow x$ \\
\hline $\operatorname{Fre}\left(\tilde{p}_{2}, 1,1\right)=2, \operatorname{Fre}\left(\tilde{p}_{2}, 2,1\right)=1$, & $\tilde{p}_{2}$ & $\hat{x}$ & $\rightarrow$ X & & \\
\hline $\begin{array}{l}\operatorname{Fre}\left(\tilde{p}_{3}, 1,1\right)=3, \operatorname{Fre}\left(\tilde{p}_{3}, 2,1\right)=1, \\
\operatorname{Fre}\left(\tilde{p}_{3}, 3,1\right)=2, \operatorname{Pre}\left(\tilde{p}_{3}, 3,2\right)=3\end{array}$ & $\tilde{p}_{3}$ & $x-$ & $\rightarrow \mathrm{X}-$ & $\rightarrow \mathrm{x}-$ & $x$ \\
\hline
\end{tabular}

Matrices $\bar{A}(1), \bar{A}(2), \bar{A}(3)$ and $\bar{A}(4)$ can then be calculated according to (20). They are respectively given (without $\varepsilon$ entries) as:

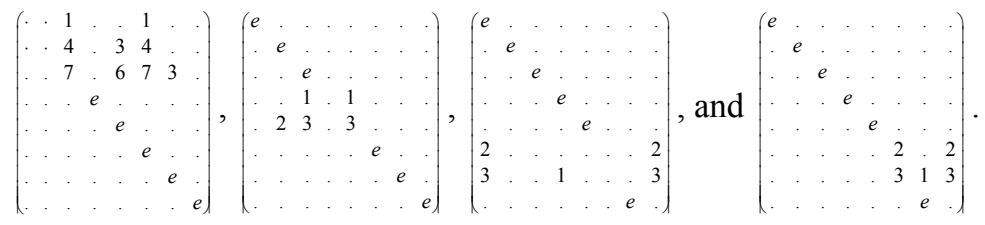

We finally calculate the monodromy matrix $\overline{\bar{A}}$. It is simply given as:

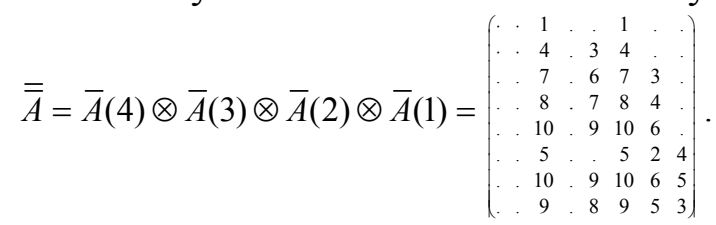

The unique finite eigenvalue of $\overline{\bar{A}}$ is equal to 9. The asymptotic production rate of $\mathcal{G}_{1}$ and $\mathcal{G}_{2}$ is therefore $\lambda_{1}=\lambda_{2}=1 / 9$ whereas the production rate of $\mathcal{G}_{3}$ is $\lambda_{3}=2 / 9$. As expected, this gives the same result as in the aforementioned works. Note however that the NCTEG of Fig. 6 is not a safe Petri net and therefore the authors in Gaubert et al. (1999) used an equivalent safe one to make possible the application of the method based on heaps-of-pieces. Finding such an equivalent safe net is not always possible especially in case of presence of input transitions. The advantage of our method is also to avoid building a heap-of-pieces automaton. We can also note that the proposed approach can be applied even with 
infinite sequences without any effect on the NCTEG structure. This is actually not the case with the method in Hillion et al. (1989) since the initial Petri net must be transformed into an equivalent TEG. Unfortunately, the resulting TEG depends dramatically on the length of the sequence and becomes very voluminous even with not long sequences (see an example in Gaubert et al. (1999)).

- Work-in-process (WIP) inventory minimization: as another application of the NCTEG-based approach and its $(\mathrm{Max},+)$ representation, we can also tackle the problem of WIP inventory minimization. It is indeed legitimate, in case of the previous manufacturing system for example, to ask question: what is the minimal number of pallets to use for each job to optimize the production rate? Such a problem has already been solved in different past investigations using linear programming as in Gaubert (1995) or Laftit et al. (1992) but only in case of systems modelled using TEGs.

By applying our $(\mathrm{Max},+)$ approach, we can solve such a problem when dealing with systems modelled using NCTEGs, not limited to TEGs.

So, to solve this problem we can begin by considering the safe case (one token in each place $p_{\text {wip }}$ ) and calculate the production rate (by calculating the eigenvalue of the monodromy matrix $\overline{\bar{A}}$ as we did before). Then, we increase progressively the number of tokens in each WIP place $p_{\text {wip }}$ of each graph on the NCTEG of Fig. 6 until the eigenvalue of the matrix $\overline{\bar{A}}$ does not change. This process was applied to example 6.1 and we got to the results of Table 3 .

Table 3: production rate (eigenvalue) and WIP inventory minimization

\begin{tabular}{|l|c|c|c|c|c|c|c|c|c|}
\hline $\begin{array}{l}\text { Case } \\
\text { wip1/ wip2 / wip3 }\end{array}$ & $1 / 1 / 1$ & $1 / 2 / 1$ & $1 / 1 / 2$ & $1 / 2 / 2$ & $2 / 1 / 1$ & $2 / 2 / 1$ & $2 / 1 / 2$ & $2 / 2 / 2$ & $3 / 3 / 3$ \\
\hline Eigenvalue & 11 & 11 & 9 & 9 & 11 & 11 & 9 & 9 & 9 \\
\hline
\end{tabular}

We can notice that the eigenvalue reaches its maximal value 11 in the safe case and does not change after getting two tokens in the WIP place of TEG $\mathcal{G}_{3}$. Its minimal value is equal to 9 . The minimal WIP inventory is therefore: one pallet for job 1, one pallet for job 2 and two pallets for job 3. Obviously, this pattern results from sequence $\sigma_{0}$ and the minimal WIP would probably change if we were to consider another sequence.

Note by the way that the approach can also be used quite easily for scheduling optimization, to maximize the production rate, by looking for the optimal basic sequence $\sigma_{0}$ that minimizes the eigenvalue of matrix $\overline{\bar{A}}$. Such a scheduling problem optimization has already been investigated in (Mayer et al. 2008) by deriving a mixed integer optimization problem. A comparison of the two methods would be an interesting outlook for future work. 


\section{Conclusion}

In this study, we extended the use of $(\mathrm{Max},+)$ algebra to Conflicting Timed Event Graphs or NCTEGs which are a natural model of a large category of practical systems involving shared resources. We proved that NCTEGs can be represented using a system of $(\mathrm{Max},+)$ Linear Time-Varying equations while the policy of resources allocation is arbitrary. In case of a cyclic NCTEG, we used the notion of monodromy to get to a standard $(\mathrm{Max},+)$ state space representation. An application on the well-known jobshop has also been presented. For future work, it would be interesting to look for the relevant modifications to bring to the proposed approach so as to relax some of the hypotheses of Section 3.1.

\section{Annexes}

Table : Notations and symbols

\begin{tabular}{|c|c|}
\hline Symbols & Meaning \\
\hline$A_{i}$ & State matrix of the $i^{t h}$ timed event graph \\
\hline$B_{i}$ & input matrix of the $i^{\text {th }}$ timed event graph \\
\hline$X_{i}$ & State vector of the $i^{\text {th }}$ timed event graph \\
\hline$U_{i}$ & Input vector of the $i^{t h}$ timed event graph \\
\hline $\mathcal{G}$ & Set of the timed event graphs of the NCTEG \\
\hline $\mathcal{G}_{i}$ & The $i^{t h}$ timed event graph of the NCTEG \\
\hline$\tilde{R}$ & Set of the places of conflict of the NCTEG \\
\hline$\tilde{p}_{j}$ & The $j^{\text {th }}$ place of conflict of the NCTEG \\
\hline$\tilde{R}_{i}$ & Set of places of conflict connected to the $i^{\text {th }}$ timed event graph \\
\hline$\breve{t}_{i j}$ & Unique transition from place $\tilde{p}_{j}$ to timed event graph $\mathcal{G}_{i}$ \\
\hline $\bar{t}_{i j}$ & Unique transition from timed event graph $\mathcal{G}_{i}$ to place $\tilde{p}_{j}$ \\
\hline$\breve{\tau}_{i j}$ & Firing time of transition $\breve{t}_{i j}$ \\
\hline $\bar{\tau}_{i j}$ & Firing time of transition $\bar{t}_{i j}$ \\
\hline
\end{tabular}




\begin{tabular}{|c|c|}
\hline$\tilde{\psi}_{j}(l)$ & Date of availability of the token of place $\tilde{p}_{j}$ for the $l^{t h}$ time \\
\hline$F_{i j}$ & Column vector relating dater $\tilde{\psi}_{j}(l)$ to state vector $X_{i}$ \\
\hline$G_{i j}$ & Line vector relating state vector $X_{i}$ to dater $\tilde{\psi}_{j}(l+1)$ \\
\hline$\sigma$ & Sequence of allocation of the shared resources \\
\hline $\operatorname{Fre}(\tilde{p}, i, k)$ & $\begin{array}{l}\text { Function giving the index of the TEG that frees resource } \tilde{p} \text {, for } k^{\text {th }} \text { time, to } \\
\text { be used subsequently by TEG } \mathcal{G}_{i}\end{array}$ \\
\hline $\bar{X}$ & Vector of vectors containing all the state vectors of the TEGs of a NCTEG \\
\hline $\bar{U}$ & Vector of vectors containing all the input vectors of the TEGs of a NCTEG \\
\hline
\end{tabular}

\section{REFERENCES}

Addad, B., Amari, S., and Lesage, J. J., (2010.a). Linear Time-Varying (Max,+) Representation of Conflicting Timed Event Graphs. $10^{\text {th }}$ Int. Workshop on Discrete Event Systems, Berlin, Germany, pp. 310-315.

Baccelli, F., Cohen, G., Olsder, G. J., and Quadrat, J. P., (1992). Synchronization and linearity: an algebra for discrete event systems, Wiley.

Baccelli, F., Foss, S., and Gaujal, B., (1996). Free choice Petri nets: an algebraic approach. IEEE Transactions on Automatic Control, 41(12), pp. 1751-1778.

Bolzern, P., Colaneri, P., and Scatolini, R. (1986). Zeros of discrete time linear periodic systems. IEEE Transaction on Automatic Control, vol. 31, pp. 1057-1058.

Boutin, O., Cottenceau, B., L'Anton, A., and Loiseau, J. J., (2009). Modeling systems with periodic routing functions in dioid (Min,+), 13th IFAC Symposium on Information Control Problems in Manufacturing, Moscow, Russia, pp. 1377-1382.

Cohen, G., Gaubert, S. and Quadrat, J. P., (1999). Max plus algebra and systems theory: where we are and where to go now, Annual Reviews in Control, vol. 23, pp. 207-219.

Correia, A., Abbas-Turki, A., Bouyekhf, and El Moudni, A. (2009) A dioid model for invariant resource sharing problems. IEEE Trans. on Systems, Man, and Cybernetics $\square$ Part A: Systems and Humans, 39(4), pp. 770-781.

Gaubert, S., and Mairesse, J., (1999). Modeling and analysis of timed Petri nets using heaps of pieces. IEEE Transactions on Automatic Control, 44(4), pp. 683-697.

Gaubert, S., (1995). Resource optimization and $(\mathrm{min},+)$ spectral theory. IEEE Transactions on Automatic Control, 40(11), pp. 1931-1934.

Hillion, H. and Proth, J. M., (1989). Performance evaluation of jobshop systems using timed event graphs, IEEE Transaction on Automatic Control, 34(1), pp. 3-9. 
Laftit, S., Proth, J. M., and Xie, X.L, (1992). Optimization of invariant criteria for event graphs, IEEE Transaction on Automatic Control, 37(6), pp. 547-555.

Lahaye, S., Boimond, J. L., and Hardouin, L., (2004) Linear periodic systems over dioids, Discrete Event Dynamic Systems, 14(2), pp 133-152.

Mayer, E., U.-U. Haus, J. Raisch and R. Weismantel (2008). Throughput-Optimal Sequences for Cyclically Operated Plants, Discrete Event Dynamic Systems 18(3), pp. 355-383.

Misra, P., (1996). Time-Invariant representation of discrete periodic systems, Automatica, 32(2), pp. 267-272.

Murata, T., (1989). Petri nets: properties analysis and applications. Proc of the IEEE, 77(4), pp. $541-580$.

Nait, S. M., Manier, M. A., El Moudni, A., and Wack, M. (2006). Petri net with conflicts and (max,plus) algebra for transportation systems, 11th IFAC Symposium on Transportation Systems, The Netherlands.

Van Den Boom, T. J. J, and De Shutter, B., (2006). Modeling and control of discrete event systems using switching Max-Plus linear systems, Control Engineering Practice, 14(10), pp. 1199-1211. 